\title{
ABORDAGEM INTERDISCIPLINAR COM OBESOS E SOBREPESOS NA ATENÇÃO PRIMÁRIA: relato de experiência
}

\author{
Andressa Názara Lucena de MELO ${ }^{1}$ \\ Fernanda Darliane Tavares de LUNA ${ }^{2}$ \\ Isaldes Stefano Vieira FERREIRA ${ }^{3}$ \\ Rosângela Vidal de NEGREIROS ${ }^{4}$ \\ Sofia de Araújo Monteiro FILOMENA ${ }^{5}$
}

\begin{abstract}
${ }^{1}$ Nutricionista. Especialista em Nutrição Materno Infantil. Prefeitura Municipal de Guarabira - PB. andressa_lucena7@hotmail.com

${ }^{2}$ Enfermeira. Mestre em Saúde Pública - UEPB. Prefeitura Municipal de Guarabira - PB. fernandarliane @ hotmail.com

${ }^{3}$ Fisioterapeuta. Especialista em Saúde da Família - UFPB. Prefeitura Municipal Guarabira - PB. isaldestefano@hotmail.com

${ }^{4}$ Enfermeira. Mestrado em Enfermagem - UFPB. Universidade Federal de Campina Grande. vidal.negreiros@gmail.com

${ }^{5}$ Fisioterapeuta. Especialista em Saúde Pública - FURNE. Hospital e Maternidade Santa Filomena - HMSF. sofiasume@hotmail.com
\end{abstract}

\section{Recebido em: 18/02/2016 - Aprovado em: 15/10/2016 - Disponibilizado em: 18/12/2016}

\section{RESUMO:}

O objetivo deste estudo foi relatar a experiência de implantação e desenvolvimento de um grupo terapêutico com indivíduos com sobrepeso e obesidade realizada em uma Unidade Básica de Saúde da Família (UBSF) de um município paraibano através de abordagem interdisciplinar com oficinas educacionais, intervencionistas e vivenciais. Foram realizadas 14 oficinas educativas e 48 encontros de atividades de práticas corporais. As oficinas educativas foram embasadas na metodologia participativa de Paulo Freire. Participaram 25 indivíduos, com idades entre 20 e 79 anos. Os principais resultados encontrados referem-se à perda de peso, melhora nos níveis pressóricos, mudanças nos hábitos alimentares e melhor qualidade de vida. Percebeu-se nesta vivência a importância da educação nutricional e da prática regular de atividade física como instrumentos de promoção, prevenção e tratamento para obesidade.

Palavras-Chave: Alimentação Saudável. Educação. Atividade Física. Qualidade de vida. Interdisciplinaridade.

\begin{abstract}
The aim of this study was to report the experience of setting up and development of a therapeutic group with overweight and obesity held in a Basic Family Health Unit (BFHU) of Paraiba municipality through an interdisciplinary approach to education, interventional and experiential workshops. They were conducted 14 educational workshops and 48 of body practical activities of meetings. Educational workshops were based on participatory methodology of Paulo Freire. Participated 25 individuals, aged between 20 and 79 years. The main results relate to weight loss, improvement in blood pressure, changes in eating habits and better quality of life. It was felt this experience the importance of nutrition education and regular physical activity as promotional tools, prevention and treatment for obesity.

KEY WORDS: Healthy Eating. Education. Physical activity. Quality of life. Interdisciplinarity.
\end{abstract}

\section{INTRODUÇÃO}

O panorama mundial e brasileiro de doenças crônicas não transmissíveis (DCNT) tem revelado um novo desafio para a saúde pública. A complexidade do perfil nutricional que se forma no país mostra a importância de um modelo de atenção à saúde que incorpore ações de promoção da saúde, prevenção e tratamento das DCNT. Neste cenário 
epidemiológico, destaca-se a obesidade por ser simultaneamente uma doença e um fator de risco para doenças como hipertensão e o diabetes (BRASIL, 2014).

A obesidade é caracterizada pelo acúmulo excessivo de gordura corporal e tornou-se um dos grandes desafios de saúde pública no Brasil e no mundo, por estar em rápida expansão e promover graves complicações na saúde. Esse agravo de saúde não é gerado por causas isoladas, influenciado por fatores genéticos, ambientais, hábitos alimentares e o sedentarismo. O tratamento é complexo e multifatorial (DIAS et. al., 2014).

No Brasil, segundo dados da última pesquisa de orçamentos familiares cerca de $15 \%$ dos adultos apresentam obesidade e a metade da população maior de 20 anos apresenta excesso de peso. Chama a atenção nessa epidemia a velocidade com que aumentou nas últimas décadas (IBGE, 2010).

No âmbito do Sistema Único de Saúde (SUS), a Política Nacional de Alimentação e Nutrição e a Política Nacional de Promoção da Saúde preveem ações específicas para a promoção da alimentação saudável. A organização das ações de promoção da alimentação saudável na Atenção Básica (AB) deve considerar os determinantes sociais da saúde, com a definição de ações técnicas e políticas, de caráter amplo e intersetorial, que respondam às necessidades de cada território, a partir de estratégias de articulação transversal entre os diversos equipamentos públicos, sociais e a comunidade (BRASIL, 2006; BRASIL, 2003).

Este cenário traz grandes desafios para a Rede de Atenção à Saúde (RAS) do SUS, em especial para a $A B$ devido a sua proximidade com o cotidiano das pessoas, do poder de compreensão da dinâmica social e dos determinantes de saúde de cada território, tornando-se local privilegiado para desenvolvimento de ações de promoção de saúde e enfrentamento do excesso de peso que acomete a população.

De acordo com dados da UBSF Naná Porpino, existe atualmente em seu território de abrangência um número elevado de indivíduos com sobrepeso e obesidade, fato que preocupa a equipe, no entanto ainda não se conseguiu atuar de forma efetiva, pois as intervenções realizadas surtiram poucos efeitos, por pequena adesão, ausência de planejamento e um projeto articulado. (UBSF NANÁ PORPINO, 2015).

Neste aspecto o presente estudo busca relatar a experiência de implantação e desenvolvimento de um grupo terapêutico com indivíduos com sobrepeso e obesidade realizada numa UBSF do município de Guarabira - PB através de abordagem interdisciplinar em oficinas educacionais, intervencionistas e vivenciais. 


\section{MATERIAIS E MÉTODOS}

Trata-se de um relato de experiência elaborado a partir de vivências em uma UBSF no município de Guarabira - PB, dentro das atividades do grupo terapêutico "Alimentação saudável e atividade física" com indivíduos com sobrepeso e obesidade, no período de Julho à Dezembro de 2015.

No total foram realizadas 14 oficinas educativas e 48 encontros de atividades de práticas corporais sob coordenação dos educadores físicos na academia da saúde e em um grupo de saúde "De bem com a vida" existente no território.

As oficinas iniciaram no mês de Julho, ocorreram de forma semanal durante o primeiro mês, posteriormente, quinzenal, sempre às quintas-feiras. As demais atividades de práticas corporais ocorreram de forma semanal, às vinculadas a academia da saúde ocorreu nas segundas-feiras e o grupo "De bem com a vida" nas quartas-feiras, ambos no período da tarde, com duração de 180 minutos, em ambiente descontraído para a realização das atividades.

O grupo foi composto por 25 usuários, sendo 23 do sexo feminino e 2 masculinos, entre 20 e 79 anos de idade, residentes da zona urbana do município, com famílias que apresentavam baixa renda mensal e escolaridade e acesso à internet limitado.

Objetivando maximizar a assiduidade e a participação continuada dos usuários e ainda despertar entusiasmo e motivação dentro do grupo, dividiu-se em dois subgrupos diferenciados pelas cores vermelho e amarelo ao qual durante os encontros realizaram programas de competição.

Ainda foi estabelecida pontuação para cada usuário, ao participar de um dos encontros recebia a pontuação de 10 “estalecas”, equivalente a sua presença no evento, que foram colocados em uma caixa para cada grupo, sendo contabilizadas no final do semestre, em passeio turístico com atividades de confraternização e encerramento na praia da baía da Traição - PB, sendo premiados os participantes com maior assiduidade e pontuação, referentes às oficinas educativas, academia da saúde e grupo de "De bem com a vida".

O modelo pedagógico adotado nas oficinas educativas baseou-se na educação conscientizadora e problematizadora de Paulo Freire, sustentada pela metodologia participativa e dialogada que favorece uma relação crítica e transformadora dos indivíduos envolvidos. A execução das oficinas foi desenvolvida de forma dinâmica a partir de situações, reflexões e verbalizações. Utilizando recursos didáticos como figuras, jogos, cartazes, músicas, fotos, desenhos e retroprojetor multimídia.

Nos encontros com os educadores físicos na academia da saúde e no grupo "De bem com a vida" desenvolveram-se atividades com exercícios de alongamento, 
aeróbicos e resistidos, utilizando bambolês, halteres, bastões, caixa de som, entre outros.

$\mathrm{O}$ primeiro encontro foi realizado no dia 09 de Julho do corrente ano no período da tarde, participaram 30 usuários. Iniciamos as atividades com a apresentação dos profissionais do NASF e da UBSF e os objetivos das oficinas e do grupo terapêutico, na ocasião realizou-se uma dinâmica objetivando socializar, descontrair e interagir, culminando com uma sondagem sobre a obesidade e suas complicações, no qual se percebeu a fragilidade do conhecimento sobre a temática, e pela presença de tabus, constrangimentos e preconceitos sendo relatados momentos de bulling ocorridos na família com denominações de "gordinha", "preguiçosa", ainda identificou-se dúvidas sobre as formas de tratamento da obesidade principalmente sobre a importância da continuidade e da reeducação alimentar aliada a prática regular de atividade física.

Durante o primeiro encontro foi solicitado o preenchimento de uma ficha de inscrição individual elaborado pelos profissionais envolvidos, objetivando caracterizar os dados sócio demográficos e clínicos dos participantes, realizando avaliação antropométrica, utilizando a balança mecânica, colocando o paciente descalço, no centro da plataforma, na posição antropométrica. Para aferição da altura, utilizou-se o estadiômetro. A medição da circunferência abdominal, braço, busto, abdômen, quadril, coxa e panturrilha direitos, utilizou-se uma fita métrica. $\mathrm{O}$ índice de massa corporal (IMC) foi calculado através da fórmula: peso/altura ${ }^{2}$, expresso em $\mathrm{Kg} / \mathrm{m}$, verificado a Pressão Arterial com esfigmomanometro e estetoscópio.

Para classificação da obesidade, foram adotados, os parâmetros estabelecidos pela Organização Mundial da Saúde (OMS), que classifica a obesidade em três níveis: o grau I que corresponde ao IMC entre $30,0 \mathrm{~kg} / \mathrm{m}^{2}$ e $34,9 \mathrm{~kg} / \mathrm{m}^{2}$ o grau II em que o IMC está entre $35,0 \mathrm{~kg} / \mathrm{m}^{2}$ e $39,9 \mathrm{~kg} / \mathrm{m}^{2} \mathrm{e}$, o grau III, ou obesidade mórbida, cujo IMC é igual ou superior a $40,0 \mathrm{~kg} / \mathrm{m}^{2}$.

$\mathrm{O}$ segundo encontro aconteceu no dia 10 de Julho os usuários foram convidados para a realização do exame de hemograma na própria UBSF, realizada por profissionais do Laboratório Municipal, objetivando avaliar as células sanguíneas e dosagens bioquímicas dos participantes e nortear as futuras condutas ao longo das atividades do grupo.

O terceiro encontro foi realizado no dia 16 de Julho, sendo abordado a temática: Obesidade, conceitos e definiçõos, complicações e tratamento. Foi apresentado vídeo educativo, e realizado escuta qualificada. Posteriormente dividimos a turma em dois grupos: equipe vermelha e a amarela a qual deveriam permanecer formadas até o final dos demais encontros, objetivando valorizar a participação e competitividade 
entre os membros, finalizamos a oficina com dinâmica de reforço da aprendizagem.

Nossa quarta oficina ocorreu no dia 23 de Julho, tratou sobre “Alimentação saudável e emagrecimento" possibilitando dar subsídios para mudanças de hábitos alimentares dos usuários, consequentemente, uma melhora na qualidade de vida, objetivando solidificar o conteúdo recebido, aprender a introduzir uma alimentação mais saudável e na medida certa.

A quinta oficina aconteceu no dia 30 de Julho sendo desenvolvido roda de conversa sobre "A importância da prática regular de atividade física e emagrecimento" com o fisioterapeuta e o educador físico.

A sexta oficina ocorreu no dia 13 de Agosto realizou-se prática de relaxamento e autopercepção corporal com a técnica de relaxamento de Jacobson, seu método desenvolve consciência corporal e relaxamento muscular progressivo, auxiliando no combate a ansiedade, estresse, dores musculares, entre outros. A sétima oficina, no dia 27 de Agosto, ocorreu conversação sobre as principais comorbidades da obesidade seguidos de momentos de reflexão, tiradúvidas e escuta qualificada.

No intuito de maximizar resultados no decorrer dos encontros foi realizado triagem dos usuários e elencado os que precisavam de atendimento médico e nutricional para que pudessem receber orientação qualificada e individualizada.
A oitava oficina aconteceu no dia 10 de Setembro, sendo realizado discussão e Roda de conversa sobre "Aspectos psicológicos no processo do emagrecimento" enfatizou-se a relevância do controle dos pensamentos e emoções no processo do emagrecimento e de uma alimentação balanceada.

O nono encontro aconteceu no dia 24 de Setembro sob a coordenação da Odontóloga da UBSF acerca da importância da mastigação no processo de emagrecimento e cuidados com a saúde bucal. Nosso décimo encontro ocorreu no dia 10 de Outubro sendo realizado "A I Caminhada Comunitária da Saúde" com os participantes do grupo e comunidade abrangente da UBSF.

O décimo primeiro encontro aconteceu no dia 22 de Outubro no período da manhã, os usuários foram convidados para a realização do exame de hemograma na própria UBSF, realizada por profissionais do Laboratório Municipal, enquanto que o décimo segundo encontro ocorreu no período da tarde sendo realizado avaliação antropométrica dos envolvidos.

No décimo terceiro encontro ocorrido no dia 19 de Novembro foi realizado oficina com o nutricionista objetivando reforçar a prática da alimentação balanceada e a escuta das dificuldades e ganhos dos usuários no decorrer do grupo. O último encontro aconteceu no dia 17 de Dezembro culminando com passeio turístico com os membros 
envolvidos, sendo realizado tarde de confraternização e encerramento das atividades neste segundo semestre de 2015 .

\section{RELATO DE EXPERIENCIA}

As atividades desenvolvidas tiveram parceria do Núcleo de Apoio à Saúde da Família - NASF composto por equipe multiprofissional: Fisioterapeuta, Psicólogo, Nutricionista, Assistente Social, Educador Físico e Fonoaudiólogo, ainda Academias de Saúde com o educador físico, Policlínica com a participação do Endocrinologista e Secretaria de Saúde e Educação.

No segundo trimestre de 2015 foram realizadas reuniões com os profissionais da UBSF Naná Porpino e membros do NASF, com intuito de traçar um diagnóstico comunitário, percebeu-se a prevalência da observância de usuários apresentando sobrepeso e/ou obesidade durante as realizações de procedimentos com os profissionais da unidade de saúde.

Diante da necessidade e inexistência de grupos educativos e intervencionistas neste respectivo território e frente à dificuldade de usuários com sobrepeso e/ou obesidade na adesão ao tratamento e da necessidade de mudanças satisfatórias no contexto da obesidade e suas complicações surgiu a ideia de implantação/criação do grupo terapêutico “Alimentação Saudável e Atividade Física". Este programa contemplou oficinas educativas e intervencionistas em grupo para discussão de temas sobre alimentação, nutrição, atividade física e saúde, com o objetivo de apoiar o atendimento coletivo, de maneira a incentivar um estilo de vida saudável, melhorar a adesão ao tratamento, diminuir fatores de risco para as comorbidades associadas à obesidade e promover qualidade de vida.

As oficinas abordaram a importância da alimentação saudável e da prática de atividades físicas regulares; obesidade e suas complicações e formas de prevenção, combate e motivação, abordagem psicológica ao indivíduo com sobrepeso e obesidade, significância da avaliação antropométrica, entre outros. Estas temáticas surgiram devido à necessidade dos usuários percebidas durante os encontros a partir das informações e vivências dos participantes.

\section{RESULTADOS E DISCUSSÃO}

Nos encontros o grupo foi composto por 25 usuários assíduos, representado por maioria do sexo feminino, com escolaridade baixa, idades entre 20 a 79 anos, quanto ao contexto socioeconômico, evidenciou-se população com baixo poder aquisitivo. A maioria dos indivíduos praticavam atividade física e (40\%) não praticavam nenhum tipo de exercício físico. Com relação ao perfil de morbidade (20\%) não relataram ter problemas de saúde e $(80 \%)$ verbalizaram ter algum dos 
seguintes agravos e/ou associados: Hipertensão Arterial Sistêmica (HAS), Diabetes Mellitus, Cardiopatia, Hipotiroidismo e depressão e/ou doenças ostiomioarticulares.

Avaliando a mensuração da pressão arterial: 5 (20\%) obtiveram níveis pressóricos compatíveis com HAS. O peso de acordo com IMC apresentou as seguintes características, 10 indivíduos com sobrepeso, 4 com obesidade grau I, 6 com obesidade grau II, 5 com excesso de peso.

Durante a avaliação cinesiofuncional pré-admissional aos grupos de práticas corporais, percebeu-se que alguns usuários apresentavam edema nos membros inferiores (MMII), pouca resistência muscular à fadiga, dispnéia, taquicardia, dormência nos membros superiores e inferiores, câimbras, dificuldades de memorização, alterações posturais, déficit em coordenação motora para realização de movimentos, dores musculares e dificuldades na marcha e níveis significativos de estresse e ansiedade.

O grupo caracterizou-se por ser colaborativo agregando informações e refletindo conjuntamente, iniciaram-se tímidos e alguns cheios de tabus quanto a aceitação do seu corpo frente a forte influência da mídia que apresenta como ideal corpos longilíneos, magros e tonificados, depois se mostraram abertos, curiosos e motivados a conhecerem os assuntos $\mathrm{e}$ demonstraram ciência na importância das discussões para a mudança de suas condutas como seres ativos no processo de mudanças de hábitos de vida.

Os encontros não tinham como intenção adotar uma postura disciplinadora e nem mesmo de determinar regras para que os participantes cumprissem rigorosamente

Durante as oficinas educativas percebeu-se um baixo grau de conhecimento sobre os temas debatidos, a maioria referiu conhecer parcialmente os principais fatores de risco e as complicações da obesidade, entendiam a importância da reeducação alimentar no processo de emagrecimento, no entanto não apresentavam conhecimento significativo, os mesmos mostraram-se surpresos quanto aos inúmeros benefícios e a importância da prática regular de atividades físicas associados a alimentação saudável.

Hábitos inadequados no padrão alimentar são fatores de risco importantes para DCNT, pois denotam uma alimentação qualitativamente inadequada, com consumo insuficiente de cereais integrais, leguminosas, raízes, tubérculos, frutas, verduras e legumes que desempenham papel fundamental na prevenção e controle destas, devido a presença de fibras alimentares, componentes antioxidantes e fitoquímicos, além de reduzir densidade calórica, promover a saciedade por aumentar o tempo de esvaziamento gástrico e diminuir a eficiência de absorção intestinal (AZEVEDO ET. AL., 2014; BARRETO, 2005). 
Desta forma, a comunidade deve ser conscientizada quanto aos riscos de uma alimentação inadequada e do sedentarismo e incentivada a mudanças de hábitos de vida, aumentando o consumo de alimentos vegetais e praticando atividades físicas.

A obesidade pode ser compreendida como agravo multifatorial, envolvendo questões biológicas, econômicas, sociais, culturais e políticas. O determinante mais imediato do acumulo excessivo de gordura, é o balanço energético positivo, na qual a quantidade de energia consumida é maior do que a quantidade gasta (BRASIL, 2014).

No que se refere ao acompanhamento deste grupo, foi necessário considerar as características da educação em saúde, tendo em vista a importância e riqueza do processo de aprendizagem que acontece através da troca de ideias, informações, habilidades e experiências.

De acordo com Marcondes (1974) A educação em saúde é um processo essencialmente ativo que envolve uma alteração no modo de pensar, sentir e agir dos indivíduos, almejando à obtenção da saúde como definida pela OMS que afirma "saúde é o estado de completo bem-estar físico, mental, e social e não simplesmente a ausência de doença e ou enfermidade". O NASF se apresenta como potencialidade para a efetivação de práticas de educação permanente em saúde, tendo em vista seus objetivos e compromissos com a troca de saberes e a mudança nos processos das equipes de Saúde (STORTI, 2012).

As ações desenvolvidas no grupo proporcionaram o aprofundamento dos conhecimentos com relação aos hábitos alimentares, atividades físicas e outras atividades de saúde interdisciplinares e integradas. Verificou-se inicialmente em se tratando de educação nutricional, que houve resistência de alguns participantes, pois implica em mudanças de hábitos alimentares, entretanto que no decorrer das reuniões notou-se algumas mudanças, como por exemplo a redução do óleo na comida, do sal, do açúcar passando a provarem com maior frequência o sabor natural das frutas e sucos, diminuição dos condimentos artificiais, do aumento da ingestão de verduras e vegetais e do respeito aos horários de refeição, antes negligenciados.

Quanto aos benefícios relacionados a adoção da atividade física dos usuários do grupo foi observado que a qualidade de vida apresentou alguns progressos tais como: a diminuição do quadro álgico, muitos relataram que estavam respirando bem melhor, dormindo com mais qualidade e menos ansiosos, voltaram a realizar suas atividades da vida diária e, pela aferição da PA, verificamos uma diminuição dos valores pressóricos e menor nível de tristeza.

Estas melhoras foram percebidas principalmente através de relatos dos usuários, grande parte afirmava durante os 
encontros da seguinte forma: "conheci mais pessoas", "me distraio mais", "sinto menos dores, “consigo fazer coisas mais rápido lá em casa", "melhorou as câimbras e diminuíram as dores", "me sinto mais forte e demoro mais a cansar", "percebo minha musculatura mais dura".

Através dos relatos acima é possível concluir que as atividades propostas contribuíram para uma melhor qualidade de vida, e influenciaram de forma positiva aspectos relacionados à promoção, prevenção e recuperação da saúde dos envolvidos, seja através de mudanças de hábitos devido a importância das atividades educativas e intervencionista, esclarecendo sobre formais mais saudáveis e orientações em geral, ou através da escuta, dando lhes oportunidade de serem ouvidos sobre seus sentimentos e experiências vivenciadas dentro e fora do grupo.

A interdisciplinaridade é a melhor forma de se trabalhar em saúde, no entanto na prática torna-se um desafio constante, não são apenas diferentes áreas do conhecimento, mas diferentes profissionais, cada um pensando a seu modo, porém todos devem atuar da forma que seja mais conveniente ao grupo. É necessário aos profissionais: flexibilidade, bom senso e compreensão, pois quem perde com os problemas que a equipe não resolve são os usuários. O trabalho interdisciplinar é bastante enriquecedor se cada profissional der sua opinião sem agredir o outro (SILVA E OLIVEIRA, 2008).

\section{CONCLUSÃO}

Aos oficinas e encontros relatados oportunizaram compartilhar os desafios, potencialidades e a necessidade de debater alimentação saudável e atividade física com a comunidade, constituindo um veículo de comunicação e espaço de educação em saúde, propício a vivência de experiências que preparam e incentivam os indivíduos para realização de hábitos saudáveis.

Os resultados encontrados confirmam a importância da terapia grupal e de uma equipe interdisciplinar, trocando informações e interligando as ações, uma vez que a obesidade é uma doença multifatorial.

Este programa orientou e monitorou o aspecto nutricional e introduziu na vida dos voluntários a atividade física, responsável não só pela queima calórica, mas como importante agente controlador do estresse e da ansiedade. A pesquisa mostra que o foco das atividades deve ser o resgate dos valores que irão motivar mudanças nos hábitos alimentares, controle dos agentes estressores e aumento da autoestima.

Os fatores de risco mais comuns para a gênese da obesidade estão mais fortemente relacionados aos hábitos de vida e alimentares dos indivíduos, ao padrão socioeconômico, nível de escolaridade, sedentarismo e 
influencias sociais. Os fatores de risco modificáveis devem ser trabalhados pelas equipes de saúde, a fim de reduzir a gênese e ou complicações trazidos pela obesidade, com implantação de grupos de autoajuda, atividades de prevenção e promoção da saúde, tais como abordagens educativas e oficinas que abordem a temática. É importante ainda destacar a relevância da realização de mobilizações, com campanhas educativas que enfatizem sobre os seus males e consequências e com a presença ativa da rede de educação, ensinando e discutindo tais temáticas desde a infância.

\section{REFERÊNCIAS}

AZEVEDO, Edynara Cristiane de Castro; DIAS, Fábia Morgana Rodrigues da Silva; DINIZ, Alcides da Silva; CABRAL, Poliana Coelho. Consumo alimentar de risco e proteção para as doenças crônicas não transmissíveis e sua associação com a gordura corporal: um estudo com funcionários da área de saúde de uma universidade pública de Recife (PE), Brasil. Ciênc. saúde coletiva vol.19 no.5 Rio de Janeiro, 2014.

BARRETO, S.M; PINHEIRO, A.R.O; SICHIERI, R; MONTEIRO, C.A; BATISTA, M.F; SHIMIDT, M. et al. Análise da estratégia global para alimentação, atividade física e saúde da Organização Mundial da Saúde. Epidemiologia e Serviços de Saúde. 2005.

BRASIL. Ministério da Saúde. Portaria no 687 MS/GM, de 30 de março de 2006. Brasília: Ministério da Saúde; 2006.
BRASIL. Ministério da Saúde. Política nacional de alimentação e nutrição. $2^{\mathrm{a}} \mathrm{ed}$. Brasília: Ministério da Saúde; 2003.

BRASIL. Ministério da Saúde. Estratégias para o cuidado da pessoa com doença crônica: obesidade. Secretaria de Atenção à Saúde. Departamento de Atenção Básica. Brasília: DF, 2014.

DIAS, Fernanda Silva; COSTA, Ariele dos Santos; NOGUEIRA, Karoline da Silva; OLIVEIRA, Osvaldinete Lopes; SANTO, Márcia Cristina Chita Espírito.

EMAGRESUS: Proposta interdisciplinar de enfrentamento da obesidade na Estratégia Saúde da Família. Cadernos de Educação, Saúde e Fisioterapia. Vol. 2. Ed. Rede Unida. ABENFISIO. Disponível em: http://revista.redeunida.org.br/ojs/index.php/c adernos-educacao-saude-

fisioterapia/article/view/166. Acesso em: 14 de Dezembro de 2015.

IBGE. Pesquisa de Orçamentos Familiares 2008-2009: antropometria e estado nutricional de crianças, adolescentes e adultos no Brasil. Rio de Janeiro: Instituto Brasileiro de Geografia e Estatística, 2010.

MARCONDES, R. S. Educação em saúde pública: conceituação, objetivos e princípios. São Paulo, Faculdade de Saúde Pública USP. Disciplina Educação em Saúde Pública, 1974.

SILVA, Sheila Aparecida da Silva; OLIVEIRA, Kilce Helena de. Interdisciplinaridade, envelhecimento e atividade física: Relato de experiência. Rev. Triang: Ens. Pesq. Ext. Uberaba - MG, v.1, n. $1,2008$.

STORTI, Moysés Martins Tosta. As Diretrizes da educação permanente em saúde nos núcleos de apoio à saúde da família - NASF. UFCS. Florianópolis - SC, 2012. 
UBSF NANÁ PORPINO. Dados Estatísticos

da Unidade Básica Saúde da Família Naná

Porpino. Guarabira - PB, 2015. 